\title{
The 10-Year Study of the Impact of Particulate Matters on Mortality in Two Transit Cities in North-Eastern Poland (PL-PARTICLES)
}

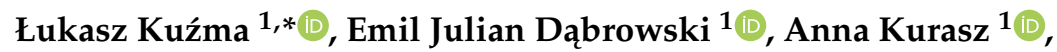 \\ Hanna Bachórzewska-Gajewska ${ }^{1,2}$ and Sławomir Dobrzycki ${ }^{1}$ \\ 1 Department of Invasive Cardiology, Medical University of Bialystok, 24A Sklodowskiej-Curie St, \\ 15-276 Bialystok, Poland; e.j.dabrowski@gmail.com (E.J.D.); annaxkurasz@gmail.com (A.K.); \\ hgajewska@op.pl (H.B.-G.); kki@umb.edu.pl (S.D.) \\ 2 Department of Clinical Medicine, Medical University of Bialystok, 24A Sklodowskiej-Curie St, \\ 15-276 Bialystok, Poland \\ * Correspondence: kuzma.lukasz@gmail.com; Tel.: +48-857468496; Fax: +48-857468828
}

Received: 20 September 2020; Accepted: 24 October 2020; Published: 27 October 2020

\begin{abstract}
The detrimental influence of air pollution on mortality has been established in a series of studies. The majority of them were conducted in large, highly polluted cities-there is a lack of studies from small, relatively clean regions. The aim was to analyze the short-term impact of particulate matters (PMs) on mortality in north-eastern Poland. Time-stratified case-crossover design was performed for mortality in years 2008-2017. Daily concentrations of $\mathrm{PM}_{2.5}\left(28.4 \mu \mathrm{g} / \mathrm{m}^{3}\right.$, interquartile range $\left.(\mathrm{IQR})=25.2\right)$ vs. $\left(12.6 \mu \mathrm{g} / \mathrm{m}^{3}, \mathrm{IQR}=9.0\right)$ and $\mathrm{PM}_{10}\left(29.0 \mu \mathrm{g} / \mathrm{m}^{3}, \mathrm{IQR}=18.0\right)$ vs. $\left(21.7 \mu \mathrm{g} / \mathrm{m}^{3}, \mathrm{IQR}=14.5\right)$ were higher in Łomża than Suwałki ( $p<0.001)$. Impact of $\mathrm{PM}_{2.5}$ on mortality was recorded in Łomża (odds ratio (OR) for IQR increase 1.061, 1.017-1.105, $p=0.06$, lag 0) and Suwałki (OR for IQR increase 1.044, 1.001-1.089, $p=0.004$, lag 0). $\mathrm{PM}_{10}$ had an impact on mortality in Łomża (OR for IQR increase 1.028, 1.000-1.058, $p=0.049$, lag 1). Cardiovascular mortality was affected by increase of $\mathrm{PM}_{2.5}$ in Łomża $(1.086,1.020-1.156$, $p=0.01)$ and Suwałki $(1.085,1.005-1.171, p=0.04) . \mathrm{PM}_{2.5}$ had an influence on respiratory mortality in Łomża $(1.163,1.021-1.380, p=0.03$, lag 1). In the whole studied region, despite differences in the air quality, the influence of PMs on mortality was observed.
\end{abstract}

Keywords: air pollution; mortality; particulate matter

\section{Introduction}

The detrimental influence of air pollution on health and mortality have been established in a series of studies by researchers all over the world [1-3]. Long-term as well as short-term exposure contributes to the development or progression of coronary artery disease, cardiac arrhythmias, heart failure, chronic obstructive pulmonary disease, and many others, with a significant impact on both life quality and life expectancy [4-7]. According to data from the Global Burden of Diseases 2017 Study, outdoor and indoor air pollution contributes to almost 5 million deaths per year [8].

Air pollution is a complex mixture of particles and gases that can be man-made (anthropogenic) as well as come from natural sources. Common air pollutants are carbon monoxide (CO), particulate matter $(\mathrm{PM})$, lead $(\mathrm{Pb})$, ozone $\left(\mathrm{O}_{3}\right)$, nitrogen dioxide $\left(\mathrm{NO}_{2}\right)$, and sulfur dioxide $\left(\mathrm{SO}_{2}\right)$. PM is typically defined by size-particles with a diameter of $10 \mu \mathrm{m}$ or less are called $\mathrm{PM}_{10}$ and with a diameter of $2.5 \mu \mathrm{m}$ or less $\mathrm{PM}_{2.5}$. We can also distinguish ultrafine particles with a diameter below $0.1 \mu \mathrm{m}$.

Over the years, changes in systemic regulations have been introduced in developed countries that have led to improvements in air quality, but even when the level of pollutants is below current targets, 
their impact on mortality is still visible. Air pollution-associated health effects are particularly pronounced in developing countries [9].

A vast amount of research focuses on highly polluted agglomerations which are an adequate place to conduct such research due to the widespread presence of an air quality monitoring system. The common belief in clean air in relatively small towns, often near rural regions, may be incorrect and have its source in the scarce number of permanent large-scale measuring stations in these areas, which is reflected in our study. Waste incineration as well as the quality of fuels used in household furnaces is not without significance. Low environmental awareness and socioeconomic conditions contribute to the deepening of the problem.

Taking this into consideration we decided to analyze clinical data with both air quality and meteorological data from two small cities over a period of 10 years.

\section{Patients and Methods}

\subsection{Study Design}

Data on mortality were collected from the National Statistical Office in Poland. The records include the information on all the deaths with the age and sex recorded in Łomża (id commune 206301) and Suwałki (id commune 200702) in the years 2008-2017. According to codes in the International Classification of Diseases-10th Revision, we extracted the data for cardiovascular-related mortality (ICD-10 from I.00 to I.99) and pulmonary-related mortality (ICD-10 from J.00 to J.99).

Standardized death rates (SDR) were calculated based on the standard European population structure [10]. The data of air pollutions and temperature were obtained from Voivodeship Inspectorate for Environmental Protection in Bialystok and the Institute of Meteorology and Water Management. In the analysis, we used the concentration of particulate matter with a diameter of $2.5 \mu \mathrm{m}$ or less $\left(\mathrm{PM}_{2.5}\right), 10 \mu \mathrm{m}$ or less $\left(\mathrm{PM}_{10}\right)$, and temperature. In Łomża city, all measurements $\left(\mathrm{PM}_{2.5}, \mathrm{PM}_{10}\right)$ were obtained from a station located in the northern part of the city-PL0151A (GPS: $53^{\circ} 18^{\prime} \mathrm{N}$, $\left.22^{\circ} 05^{\prime} \mathrm{E}\right)$. The temperature measurements were obtained for years 2008-2011 from station ID 253210210 (GPS: 53 $39^{\prime} \mathrm{N}, 21^{\circ} 34^{\prime} \mathrm{E}$ ), for 2011-2015 (ID 253220280; GPS: 53 $24^{\prime} \mathrm{N}, 22^{\circ} 15^{\prime} \mathrm{E}$ ) and station 253220330 (GPS $53^{\circ} 21^{\prime} \mathrm{N}, 22^{\circ} 10^{\prime} \mathrm{E}$ ) for 2016 and 2017. In Suwałki, the measurements of air pollution were obtained from station PL0152A (GPS: $54^{\circ} 12^{\prime} \mathrm{N}, 22^{\circ} 93^{\prime} \mathrm{E}$ ), the temperature from the station ID 354220195 (GPS: $54^{\circ} 13^{\prime} \mathrm{N}, 22^{\circ} 95^{\prime} \mathrm{E}$ ) (Figure 1).

The $24 \mathrm{~h}$ mean daily values of physical data were used for statistical analysis.

The exceedance of air pollution norms was determined based on the World Health Organization guidelines concerning air quality. The 24-h concentrations recommended by the WHO are $50 \mu \mathrm{g} / \mathrm{m}^{3}$, $25 \mu \mathrm{g} / \mathrm{m}^{3}$ for $\mathrm{PM}_{10}, \mathrm{PM}_{2.5}$, respectively [11]. The study material lacked about $14.1 \%$ in Łomża and $24.49 \%$ in Suwałki. Days with missing data were excluded from the analysis.

\subsection{Region's Characteristics}

Both of the analyzed cities are located in Podlaskie Voivodeship-north-eastern part of Poland widely known as the Green Lungs of Poland. The name of the region is derived from the surrounding of national parks, lack of factories, and relatively low industrialization. However, despite their unique location, the characteristics of both cities contribute to an increased level of air pollution. Suwałki and Łomża take an important part in the transit traffic from Northern and Eastern Europe to Central Europe and during the study, neither of the cities had ring roads. Both cities have a similar population, percentages of people over 65 years old with $15.1 \%$ in Suwałki and $16.7 \%$ in Łomża, and femininity ratio of 109 in Suwałki and 110 in Łomża. 


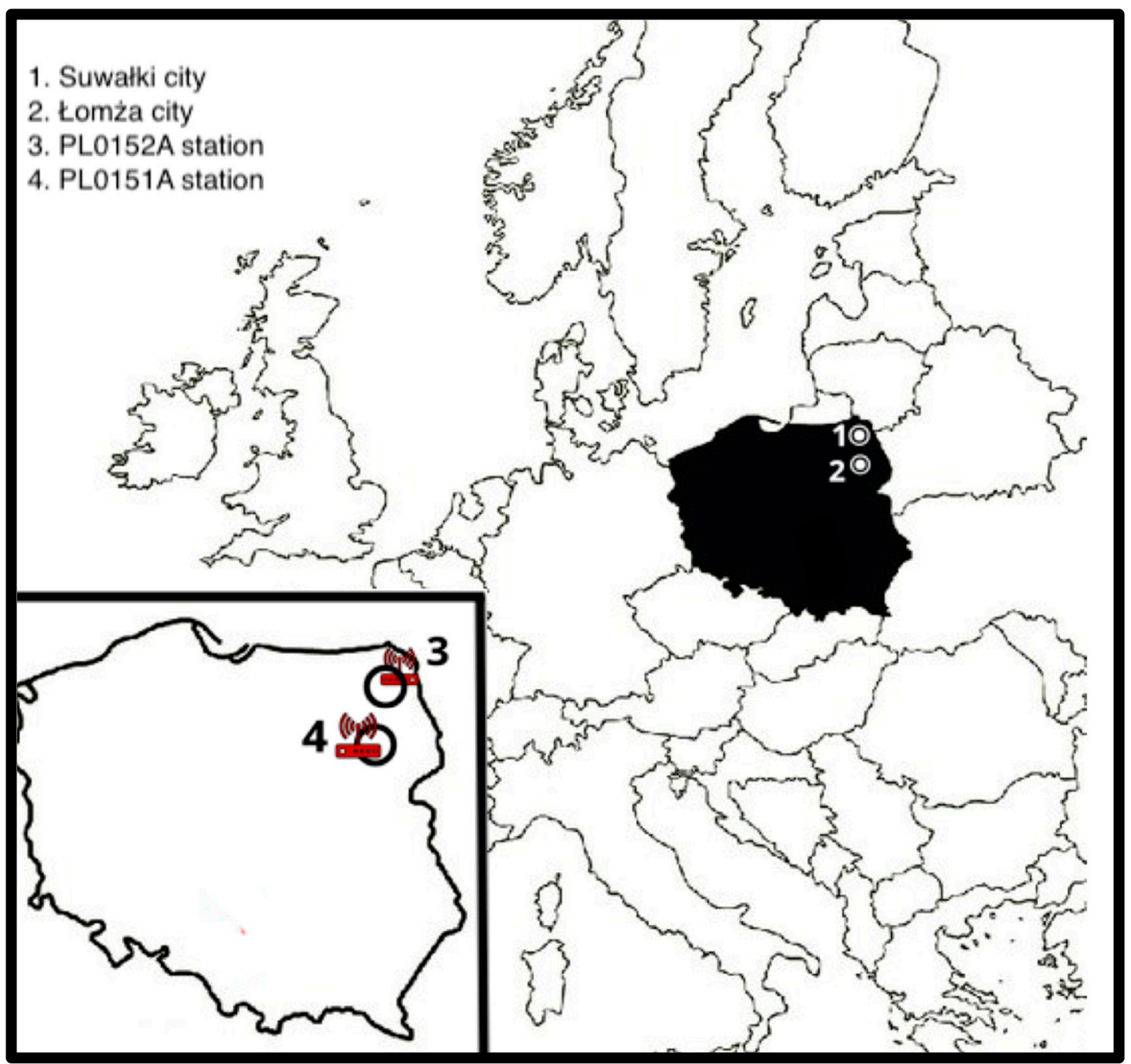

Figure 1. Characteristics of the studied cites.

\subsection{Statistical Analysis}

The distribution of variables was evaluated using the Kolmogorov-Smirnov test. Variables were expressed as mean values with standard deviation. A two-tailed t-test was used for comparative analysis. Spearman correlation test was applied for evaluating the relationships between the levels of PMs and temperature. Results are reported as $\mathrm{r}$ score and plots.

To assess the effect of particulate matter on mortality we used a time-stratified case-crossover study design $[12,13]$. The day of death was defined as the case period, while control periods included all days that were from the same day of the week in the same month as the case period. Because of high collinearity, to minimize that effect, each air pollutant was modeled individually. Separate models were created for temperature.

The association between PMs and the occurrence of deaths was estimated by odds ratios (ORs) with $95 \%$ confidence intervals (CIs) using conditional logistic regression (CLR). Meteorological data including temperature during the same lag period were used as covariates in the CLR model. We used a natural cubic spline with 5 degrees of freedom for the temperature-mortality function. We made separated models for total mortality, cardiovascular mortality and pulmonary mortality.

Results are reported as OR associated with an increase in interquartile range (IQR) at days with lag from 0 to 2.

The differences in the odds ratio between cities were calculated according to the Altman method [14]. The results were presented as ratios of OR (ROR) and CI from the 5th to the 95th percentile. 
All analyses were performed using MS Excel (Microsoft, 2020, version 16.40, Redmond, WA, USA) and XL Stat (Addinsoft, 2020, version 2020.03.01, New York, NY, USA). The study protocol conformed to the ethical guidelines of the 1975 Declaration of Helsinki, STROBE guidelines [15], and was approved by the local bioethics committee of the Medical University of Bialystok (Approval No. R-1-002/18/2019) and registered in the database of clinical studies www.clinicaltrials.gov (Identifier: NCT04541498).

The two-tailed $p$-value $<0.05$ was considered statistically significant.

\section{Results}

From 2008 to 2017 we recorded 7486 deaths in Suwałki and 8082 in Łomża. The male sex was dominant in both of the cities- $54.1 \%$ in Suwałki, $54.5 \%$ in Łomża. The mean age of deaths in Suwałki was 71.7 years $(\mathrm{SD}$ (standard deviation) $=16.6)$, in Łomża-72.7 years $(\mathrm{SD}=15.7),(p<0.001)$. The crude death rate (CDR) (1288.1 per 100,000 population/year) as well as standardized death rate (SDR) (1944.6 per 100,000 population/year) was the highest in Łomża (Table 1).

Table 1. Mortality data in the studied cities.

\begin{tabular}{cccc}
\hline & Suwałki & Lomża & $p$ \\
\hline Total deaths, N & 7486 & 8082 & \\
\hline Male, $\%(\mathrm{~N})$ & $54.1(4055)$ & $54.5(4408)$ & 0.640 \\
\hline Mean age (SD) & $71.7(16.6)$ & $72.7(15.7)$ & $<0.001$ \\
\hline CDR $(100,000$ population/year) & 1079.5 & 1288.1 & $<0.001$ \\
\hline SDR $(100,000$ population/year $)$ & 1638.1 & 1944.6 & $<0.001$ \\
\hline SD, standard deviation; CDR, crude death rate; SDR, standardized death rate.
\end{tabular}

In Suwałki, more prevalent causes of death were malignant neoplasm of bronchus and lung $(8.0 \%$ $(\mathrm{N}=597)$ vs. $6.4 \%(\mathrm{~N}=518), p<0.001)$, malignant neoplasm of breast $(2.1 \%(\mathrm{~N}=157)$ vs. $1.6 \%(\mathrm{~N}=127)$; $p=0.014)$, hypertensive heart disease $(2.2 \%(\mathrm{~N}=168)$ vs. $1.8 \%(\mathrm{~N}=144) ; p=0.040)$, and unspecified heart disease $(5.0 \%(\mathrm{~N}=372)$ vs. $3.5 \%(\mathrm{~N}=285) ; p<0.001)$. Heart failure $(3.4 \%(\mathrm{~N}=276)$ vs. $1.9 \%$ $(\mathrm{N}=141) ; p<0.001)$, cerebral infarction $(9.2 \%(\mathrm{~N}=744)$ vs. $5.8 \%(\mathrm{~N}=432), p<0.001)$, myocardial infarction $(3.9 \%(\mathrm{~N}=315)$ vs. $3.1 \%(\mathrm{~N}=232) ; p=0.007)$, and chronic obstructive pulmonary disease $(3.2 \%(\mathrm{~N}=258)$ vs. $2.6 \%(\mathrm{~N}=193) ; p=0.022)$ were more frequent causes of deaths in Łomża (Table 2).

Over the observation time, daily mean concentrations of $\mathrm{PM}_{2.5}$ and $\mathrm{PM}_{10}$ were analyzed as well as the mean levels of temperature. In each of the analyzed years, concentrations of $\mathrm{PM}_{2.5}$ and $\mathrm{PM}_{10}$ were higher in Łomża. The daily concentrations of $\mathrm{PM}_{2.5}\left(28.4 \mu \mathrm{g} / \mathrm{m}^{3}, \mathrm{SD}=24.3, \mathrm{IQR}=25.2\right)$ vs. (12.6 $\left.\mu \mathrm{g} / \mathrm{m}^{3}, \mathrm{SD}=9.2, \mathrm{IQR}=9.0\right)$ and $\mathrm{PM}_{10}\left(29.0 \mu \mathrm{g} / \mathrm{m}^{3}, \mathrm{SD}=19.4, \mathrm{IQR}=18.0\right) \mathrm{vs} .\left(21.7 \mu \mathrm{g} / \mathrm{m}^{3}, \mathrm{SD}=14.0\right.$, IQR $=14.5$ were higher in Łomża than Suwałki $(p<0.001)$ (Table 3$)$. The limit of the daily mean given by the WHO guidelines for $\mathrm{PM}_{2.5}$ in Łomża and Suwałki was exceeded on $40.7 \%$ and $8.4 \%$ of days, respectively. The daily WHO upper limit for $\mathrm{PM}_{10}$ was exceeded on $9.8 \%$ of days in Łomża and $4.2 \%$ in Suwałki. The daily mean temperature in Łomża was $7.9^{\circ} \mathrm{C}(\mathrm{SD}=8.8)$ and in Suwałki it was $7.4^{\circ} \mathrm{C}$ (8.8) (Table 3 and Figure 2).

A strong positive correlation was found between concentration of particulate matters- $\mathrm{PM}_{2.5}$ vs. $\mathrm{PM}_{10}(\mathrm{r}=0.668, p<0.001)$ in Suwałki. A strong negative correlation was observed in Łomża between $\mathrm{PM}_{2.5}$ vs. temperature $(\mathrm{r}=-0.608, p<0.001)$ alongside moderate positive $\mathrm{PM}_{2.5}$ vs. $\mathrm{PM}_{10}$ correlation $(\mathrm{r}=0.518, p<0.001)($ Table 4$)$. 
Table 2. Cause-specific mortality in studied cities.

\begin{tabular}{|c|c|c|c|}
\hline & Suwałki & Łomża & $p$ \\
\hline All, \% (N) & $100(7486)$ & $100(8082)$ & N/A \\
\hline Cardiovascular deaths, \% (N) & $36.4(2724)$ & $41.2(3328)$ & $<0.001$ \\
\hline Pulmonary deaths, $\%(\mathrm{~N})$ & $7.3(549)$ & $6.5(528)$ & $<0.001$ \\
\hline Chronic ischemic heart disease, $\%(\mathrm{~N})$ & $8.5(633)$ & $9.1(733)$ & 0.176 \\
\hline Cerebral infarction, $\%(\mathrm{~N})$ & $5.8(432)$ & $9.2(744)$ & $<0.001$ \\
\hline Heart disease-unspecified, $\%(\mathrm{~N})$ & $5.0(372)$ & $3.5(285)$ & $<0.001$ \\
\hline Myocardial infarction, $\%(\mathrm{~N})$ & $3.1(232)$ & $3.9(315)$ & 0.007 \\
\hline Intracerebral hemorrhage, $\%(\mathrm{~N})$ & $2.3(174)$ & $2.7(219)$ & 0.126 \\
\hline Hypertensive heart disease, $\%(\mathrm{~N})$ & $2.2(168)$ & $1.8(144)$ & 0.040 \\
\hline Heart failure, $\%(\mathrm{~N})$ & $1.9(141)$ & $3.4(276)$ & $<0.001$ \\
\hline Malignant neoplasm of bronchus and lung, \% (N) & $8.0(597)$ & $6.4(518)$ & $<0.001$ \\
\hline Instantaneous death, $\%(\mathrm{~N})$ & $2.6(196)$ & $2.8(225)$ & 0.524 \\
\hline Chronic obstructive pulmonary disease, \% (N) & $2.6(193)$ & $3.2(258)$ & 0.022 \\
\hline Pneumonia, \% (N) & $2.5(188)$ & $2.3(183)$ & 0.313 \\
\hline Diabetes mellitus, \% (N) & $2.1(160)$ & $2.6(206)$ & 0.090 \\
\hline Malignant neoplasm of breast, \% (N) & $2.1(157)$ & $1.6(127)$ & 0.014 \\
\hline Malignant neoplasm of colon, \% (N) & $2.0(152)$ & $1.9(152)$ & 0.121 \\
\hline Malignant neoplasm of prostate, \% (N) & $2.0(148)$ & $1.7(137)$ & 0.190 \\
\hline Senility, \% (N) & $1.9(139)$ & $2.2(176)$ & 0.155 \\
\hline Suicide, $\%(\mathrm{~N})$ & $1.8(132)$ & $0.8(62)$ & $<0.001$ \\
\hline Atherosclerosis, \% (N) & $1.7(125)$ & $1.8(146)$ & 0.515 \\
\hline Malignant neoplasm of gastric, $\%(\mathrm{~N})$ & $1.6(116)$ & $1.4(115)$ & 0.514 \\
\hline Other, \% (N) & $40.5(3031)$ & $37.9(3061)$ & $<0.001$ \\
\hline
\end{tabular}

N/A; not applicable.

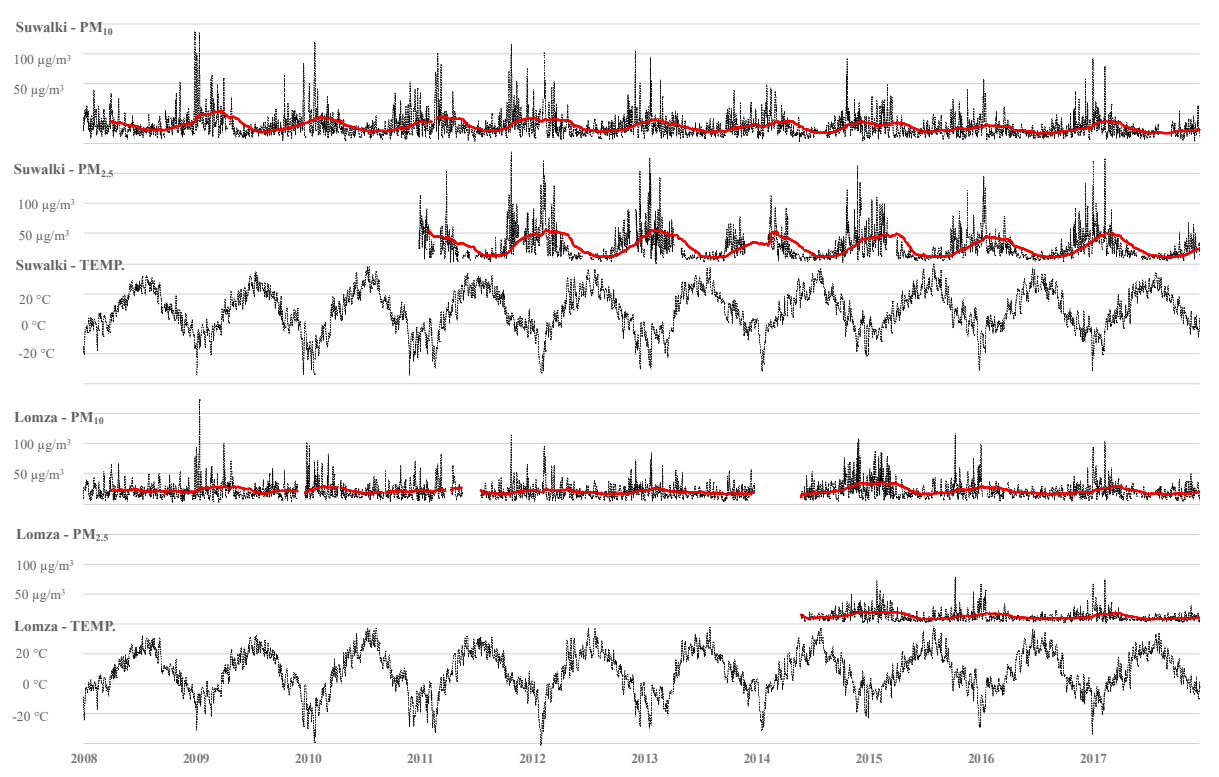

Figure 2. Panel chart. Changes in the concentrations of air pollutants and temperature in studied cities for analyzed period (the red line represents changes in the quartile of the year). 
Table 3. Statistics for daily concentrations of particulate matters and temperature.

\begin{tabular}{|c|c|c|c|c|c|c|c|c|c|}
\hline \multirow{3}{*}{$\begin{array}{l}\text { Variables } \\
\text { h observation; N, (\%) }\end{array}$} & \multicolumn{3}{|c|}{$\mathbf{P M}_{2.5} \mu \mathrm{g} / \mathrm{m}^{3}$} & \multicolumn{3}{|c|}{$\mathbf{P M}_{10} \mu \mathrm{g} / \mathrm{m}^{3}$} & \multicolumn{3}{|c|}{ Temp. ${ }^{\circ} \mathrm{C}$} \\
\hline & \multicolumn{2}{|c|}{ Suwałki Łomża } & \multirow{2}{*}{$\begin{array}{c}p \\
<0.001\end{array}$} & \multicolumn{2}{|c|}{ Suwałki Łomża } & \multirow{2}{*}{$\begin{array}{c}p \\
<0.001\end{array}$} & \multicolumn{2}{|c|}{ Suwałki Łomża } & \multirow{2}{*}{$\begin{array}{c}p \\
\text { N/A }\end{array}$} \\
\hline & $\begin{array}{c}1309 \\
(35.8)\end{array}$ & $\begin{array}{c}2230 \\
(61.1)\end{array}$ & & $\begin{array}{c}3313 \\
(90.7)\end{array}$ & $\begin{array}{c}3533 \\
(96.7)\end{array}$ & & $\begin{array}{l}3653 \\
(100)\end{array}$ & $\begin{array}{l}3653 \\
(100)\end{array}$ & \\
\hline 2008; mean/day (SD) & N/D & $\mathrm{N} / \mathrm{D}$ & N/A & $\begin{array}{c}21.5 \\
(11.6)\end{array}$ & $\begin{array}{c}31.2 \\
(19.1)\end{array}$ & $<0.001$ & $\begin{array}{c}8.0 \\
(7.2)\end{array}$ & $\begin{array}{c}8.5 \\
(7.3)\end{array}$ & 0.346 \\
\hline 2009; mean/day (SD) & $\mathrm{N} / \mathrm{D}$ & $\mathrm{N} / \mathrm{D}$ & N/A & $\begin{array}{c}23.7 \\
(18.2)\end{array}$ & $\begin{array}{c}34.1 \\
(25.1)\end{array}$ & $<0.001$ & $\begin{array}{c}6.9 \\
(8.6)\end{array}$ & $\begin{array}{c}7.2 \\
(8.6)\end{array}$ & 0.559 \\
\hline 2010; mean/day (SD) & $\mathrm{N} / \mathrm{D}$ & $\mathrm{N} / \mathrm{D}$ & N/A & $\begin{array}{c}22.1 \\
(13.3)\end{array}$ & $\begin{array}{c}29.9 \\
(19.8)\end{array}$ & $<0.001$ & $\begin{array}{c}6.3 \\
(10.7)\end{array}$ & $\begin{array}{c}6.5 \\
(10.5)\end{array}$ & 0.754 \\
\hline 2011; mean/day (SD) & $\mathrm{N} / \mathrm{D}$ & $\begin{array}{l}33.02 \\
(25.6)\end{array}$ & N/A & $\begin{array}{c}21.4 \\
(14.2)\end{array}$ & $\begin{array}{c}34.0 \\
(23.8)\end{array}$ & $<0.001$ & $\begin{array}{c}7.4 \\
(9.0)\end{array}$ & $\begin{array}{c}8.1 \\
(8.9)\end{array}$ & 0.346 \\
\hline 2012; mean/day (SD) & $\mathrm{N} / \mathrm{D}$ & $\begin{array}{c}33.2 \\
(29.4)\end{array}$ & N/A & $\begin{array}{c}20.2 \\
(12.8)\end{array}$ & $\begin{array}{c}29.9 \\
(20.1)\end{array}$ & $<0.001$ & $\begin{array}{c}6.6 \\
(9.8)\end{array}$ & $\begin{array}{c}7.3 \\
(9.9)\end{array}$ & 0.350 \\
\hline 2013; mean/day (SD) & $\mathrm{N} / \mathrm{D}$ & $\begin{array}{c}27.9 \\
(24.7)\end{array}$ & N/A & $\begin{array}{c}19.1 \\
(11.2)\end{array}$ & $\begin{array}{c}27.1 \\
(15.7)\end{array}$ & $<0.001$ & $\begin{array}{c}7.2 \\
(9.1)\end{array}$ & $\begin{array}{c}7.7 \\
(9.0)\end{array}$ & 0.535 \\
\hline 2014; mean/day (SD) & $\begin{array}{l}15.1 \\
(8.7)\end{array}$ & $\begin{array}{c}28.0 \\
(24.5)\end{array}$ & $<0.001$ & $\begin{array}{c}25.9 \\
(16.8)\end{array}$ & $\begin{array}{c}29.4 \\
(18.0)\end{array}$ & 0.007 & $\begin{array}{c}7.8 \\
(8.8)\end{array}$ & $\begin{array}{c}8.2 \\
(8.7)\end{array}$ & 0.551 \\
\hline 2015; mean/day (SD) & $\begin{array}{c}13.2 \\
(10.8)\end{array}$ & $\begin{array}{c}26.6 \\
(21.8)\end{array}$ & $<0.001$ & $\begin{array}{c}24.22 \\
(16.72)\end{array}$ & $\begin{array}{c}26.1 \\
(15.6)\end{array}$ & 0.004 & $\begin{array}{c}8.3 \\
(7.4)\end{array}$ & $\begin{array}{c}9.1 \\
(7.7)\end{array}$ & 0.218 \\
\hline 2016; mean/day (SD) & $\begin{array}{c}11.6 \\
(8.02)\end{array}$ & $\begin{array}{c}25.9 \\
(21.2)\end{array}$ & $<0.001$ & $\begin{array}{c}19.3 \\
(10.0)\end{array}$ & $\begin{array}{c}23.6 \\
(14.5)\end{array}$ & $<0.001$ & $\begin{array}{c}7.6 \\
(8.5)\end{array}$ & $\begin{array}{c}8.2 \\
(8.4)\end{array}$ & 0.272 \\
\hline 2017; mean/day (SD) & $\begin{array}{l}11.4 \\
(8.5)\end{array}$ & $\begin{array}{c}25.6 \\
(21.8)\end{array}$ & $<0.001$ & $\begin{array}{c}21.0 \\
(13.2)\end{array}$ & $\begin{array}{c}24.8 \\
(16.7)\end{array}$ & $<0.001$ & $\begin{array}{c}7.5 \\
(7.9)\end{array}$ & $\begin{array}{c}8.4 \\
(8.1)\end{array}$ & 0.108 \\
\hline Total; mean/day (SD) & $\begin{array}{l}12.6 \\
(9.2)\end{array}$ & $\begin{array}{c}28.4 \\
(24.3)\end{array}$ & $<0.001$ & $\begin{array}{c}21.7 \\
(14.0)\end{array}$ & $\begin{array}{c}29.0 \\
(19.4)\end{array}$ & $<0.001$ & $\begin{array}{c}7.4 \\
(8.8)\end{array}$ & $\begin{array}{c}7.9 \\
(8.8)\end{array}$ & 0.009 \\
\hline 1st quartile & 6.6 & 12.2 & $<0.001$ & 12.5 & 16.9 & $<0.001$ & 1.2 & 1.6 & 0.009 \\
\hline Daily median & 9.9 & 20.0 & $<0.001$ & 18.1 & 24.0 & $<0.001$ & 7.2 & 7.7 & 0.009 \\
\hline 3rd quartile & 15.5 & 37.4 & $<0.001$ & 27.0 & 35.0 & $<0.001$ & 14.6 & 15.3 & 0.009 \\
\hline IQR & 9.0 & 25.2 & $<0.001$ & 14.5 & 18.0 & $<0.001$ & 13.4 & 13.7 & 0.009 \\
\hline $\begin{array}{l}\text { Exceed daily mean WHO } \\
\text { guideline; } \mathrm{N}(\%)\end{array}$ & $\begin{array}{l}110 \\
(8.4)\end{array}$ & $\begin{array}{c}908 \\
(40.7)\end{array}$ & $<0.001$ & $\begin{array}{c}139 \\
(4.2)\end{array}$ & $\begin{array}{l}345 \\
(9.8)\end{array}$ & $<0.001$ & N/A & N/A & N/A \\
\hline
\end{tabular}

IRQ, interquartile range; N/A, not applicable, N/D, no data; $\mathrm{PM}_{2.5}$, particulate matter with a diameter of $2.5 \mu \mathrm{m}$ or less, $\mathrm{PM}_{10}$, particulate matter with a diameter of $10 \mu \mathrm{m}$ or less; SD, standard deviation; Temp., temperature; WHO, World Health Organization.

Table 4. Spearman correlation between psychical variables (left part of the chart-Suwałki, right-Łomża).

\begin{tabular}{ccc}
\hline $\begin{array}{c}\mathbf{P M}_{2.5} \\
\mu \mathrm{g} / \mathrm{m}^{3}\end{array}$ & $\mathrm{r}=0.518 ; p<0.001$ & $\mathrm{r}=-0.608 ; p<0.001$ \\
$\mathrm{r}=0.668 ; p<0.001$ & $\begin{array}{c}\mathbf{P M}_{10} \\
\mu \mathrm{g} / \mathbf{m}^{3}\end{array}$ & $\mathrm{r}=-0.303 ; p<0.001$ \\
\hline $\mathrm{r}=-0.268 ; p<0.001$ & $\mathrm{r}=-0.243 ; p<0.001$ & $\begin{array}{c}\text { Temperature } \\
{ }^{\circ} \mathbf{C}\end{array}$ \\
\hline
\end{tabular}

The impact of $\mathrm{PM}_{2.5}$ on total mortality was recorded in Suwałki (OR for IQR increase 1.044, 95\% CI 1.001-1.0.89, $p=0.04$ ) and Łomża (OR for IQR increase 1.061, 95\%CI 1.017-1.105, $p=0.006$ ). The effect of $\mathrm{PM}_{10}$ was observed in Łomża on lag 1 (OR for IQR increase 1.028, 95\% CI 1.000-1.058, $p=0.049$ ) and lag 2 (OR for IQR increase 1.030, 95\% CI 1.001-1.060, $p=0.04$ ). In case of Suwałki the effect of $\mathrm{PM}_{10}$ was observed at lag $2 \mathrm{OR}=1.034(95 \%$ CI 1.005-1.064, $p=0.02)$. 
The effect of PMs on cardiovascular mortality was noted in Suwałki on lag 0 OR $=1.085$ (95\% CI 1.005-1.171, $p=0.037$ ) for $\mathrm{PM}_{2.5}$ and $\mathrm{OR}=1.056$ (95\% CI 1.006-1.07, $p=0.03$ ) for $\mathrm{PM}_{10}$. Moreover, in Łomża, the effect of $\mathrm{PM}_{2.5}$ was also noted (OR 1.086, 95\% CI 1.020-1.156, $p=0.01$, lag 0).

In the analysis of pulmonary mortality, only the effect of $\mathrm{PM}_{10}$ on lag 1 was noted in Łomża $(\mathrm{OR}=1.16395 \% \mathrm{Cl} 1.021-1.380)$. No differences in the impact of air pollution on mortality were observed between both cities (Table 5).

Table 5. Time-stratified case-crossover model for study population. The odds ratio of mortality with interquartile-range increase in exposure to air pollutants and temperature.

\begin{tabular}{|c|c|c|c|c|c|c|c|c|}
\hline & \multicolumn{2}{|c|}{ Variables } & \multicolumn{2}{|c|}{ Suwałki } & \multicolumn{2}{|c|}{ Łomża } & \multicolumn{2}{|c|}{ Ratio of Odds Ratio } \\
\hline & & & OR $(95 \% \mathrm{CI})$ & $p$ & OR $(95 \% \mathrm{CI})$ & $p$ & $\begin{array}{l}\text { ROR }(95 \% \\
\text { CI) }\end{array}$ & $p$ \\
\hline \multirow{6}{*}{ Total mortality } & \multirow{2}{*}{ LAG 0} & $\mathrm{PM}_{2.5}$ & $\begin{array}{c}1.044 \\
(1.001-1.089)\end{array}$ & 0.04 & $\begin{array}{c}1.061 \\
(1.017-1.105)\end{array}$ & 0.006 & $\begin{array}{c}0.984 \\
(0.972-1.044) \\
\end{array}$ & 0.29 \\
\hline & & $\mathrm{PM}_{10}$ & $\begin{array}{c}1.024 \\
(0.995-1.054)\end{array}$ & 0.10 & $\begin{array}{c}1.018 \\
(0.991-1.047)\end{array}$ & 0.21 & $\begin{array}{c}1.005 \\
(0.966-1.047)\end{array}$ & 0.38 \\
\hline & \multirow{2}{*}{ LAG 1} & $\mathrm{PM}_{2.5}$ & $\begin{array}{c}1.027 \\
(0.981-1.075)\end{array}$ & 0.27 & $\begin{array}{c}1.029 \\
(0.988-1.071)\end{array}$ & 0.17 & $\begin{array}{c}0.998 \\
(0.339-1.061)\end{array}$ & 0.48 \\
\hline & & $\mathrm{PM}_{10}$ & $\begin{array}{c}1.006 \\
(0.978-1.036)\end{array}$ & 0.66 & $\begin{array}{c}1.028 \\
(1.000-1.058)\end{array}$ & 0.049 & $\begin{array}{c}0.977 \\
(0.939-1.017)\end{array}$ & 0.14 \\
\hline & \multirow{2}{*}{ LAG 2} & $\mathrm{PM}_{2.5}$ & $\begin{array}{c}1.005 \\
(0.961-1.052)\end{array}$ & 0.83 & $\begin{array}{c}1.036 \\
(0.995-1.078)\end{array}$ & 0.82 & $\begin{array}{c}0.971 \\
(0.913-1.032)\end{array}$ & 0.17 \\
\hline & & $\mathrm{PM}_{10}$ & $\begin{array}{c}1.034 \\
(1.005-1.064) \\
\end{array}$ & 0.02 & $\begin{array}{c}1.030 \\
(1.001-1.060) \\
\end{array}$ & 0.04 & $\begin{array}{c}1.004 \\
(0.965-1.045) \\
\end{array}$ & 0.43 \\
\hline \multirow{6}{*}{$\begin{array}{c}\text { Cardiovascular } \\
\text { mortality }\end{array}$} & \multirow{2}{*}{ LAG 0} & $\mathrm{PM}_{2.5}$ & $\begin{array}{c}1.085 \\
(1.005-1.171)\end{array}$ & 0.04 & $\begin{array}{c}1.086 \\
(1.020-1.156)\end{array}$ & 0.01 & $\begin{array}{c}0.999 \\
(0.905-1.103)\end{array}$ & 0.50 \\
\hline & & $\mathrm{PM}_{10}$ & $\begin{array}{c}1.056 \\
(1.006-1.107)\end{array}$ & 0.03 & $\begin{array}{c}1.022 \\
(0.979-1.067)\end{array}$ & 0.33 & $\begin{array}{c}1.033 \\
(0.972-1.098)\end{array}$ & 0.15 \\
\hline & \multirow{2}{*}{ LAG 1} & $\mathrm{PM}_{2.5}$ & $\begin{array}{c}1.034 \\
(0.957-1.116)\end{array}$ & 0.39 & $\begin{array}{c}1.029 \\
(0.967-1.095)\end{array}$ & 0.37 & $\begin{array}{c}1.005 \\
(0.910-1.109)\end{array}$ & 0.46 \\
\hline & & $\mathrm{PM}_{10}$ & $\begin{array}{c}1.004 \\
(0.957-1.054)\end{array}$ & 0.86 & $\begin{array}{c}1.034 \\
(0.991-1.080)\end{array}$ & 0.13 & $\begin{array}{c}0.971 \\
(0.909-1.036)\end{array}$ & 0.19 \\
\hline & \multirow{2}{*}{ LAG 2} & $\mathrm{PM}_{2.5}$ & $\begin{array}{c}1.014 \\
(0.939-1.094)\end{array}$ & 0.73 & $\begin{array}{c}0.992 \\
(0.932-1.056)\end{array}$ & 0.80 & $\begin{array}{c}0.981 \\
(0.898-1.071)\end{array}$ & 0.33 \\
\hline & & $\mathrm{PM}_{10}$ & $\begin{array}{c}1.025 \\
(0.977-1.076)\end{array}$ & 0.31 & $\begin{array}{c}1.008 \\
(0.965-1.053)\end{array}$ & 0.72 & $\begin{array}{c}1.017 \\
(0.952-1.085)\end{array}$ & 0.31 \\
\hline \multirow{6}{*}{$\begin{array}{l}\text { Pulmonary } \\
\text { mortality }\end{array}$} & \multirow{2}{*}{ LAG 0} & $\mathrm{PM}_{2.5}$ & $\begin{array}{c}1.161 \\
(0.987-1.365)\end{array}$ & 0.072 & $\begin{array}{c}1.130 \\
(0.967-1.320)\end{array}$ & 0.12 & $\begin{array}{c}1.027 \\
(0.821-1.286)\end{array}$ & 0.41 \\
\hline & & $\mathrm{PM}_{10}$ & $\begin{array}{c}1.023 \\
(0.916-1.141)\end{array}$ & 0.68 & $\begin{array}{c}1.011 \\
(0.906-1.128)\end{array}$ & 0.87 & $\begin{array}{c}1.012 \\
(0.866-1.181)\end{array}$ & 0.44 \\
\hline & \multirow{2}{*}{ LAG 1} & $\mathrm{PM}_{2.5}$ & $\begin{array}{c}1.040 \\
(0.885-1.221) \\
\end{array}$ & 0.64 & $\begin{array}{c}1.163 \\
(1.021-1.380)\end{array}$ & 0.03 & $\begin{array}{c}0.894 \\
(0.717-1.115) \\
\end{array}$ & 0.16 \\
\hline & & $\mathrm{PM}_{10}$ & $\begin{array}{c}0.979 \\
(0.879-1.091)\end{array}$ & 0.69 & $\begin{array}{c}1.013 \\
(0.904-1.135)\end{array}$ & 0.82 & $\begin{array}{c}0.966 \\
(0.826-1.131)\end{array}$ & 0.33 \\
\hline & \multirow{2}{*}{ LAG 2} & $\mathrm{PM}_{2.5}$ & $\begin{array}{c}0.898 \\
(0.759-1.062)\end{array}$ & 0.21 & $\begin{array}{c}1.073 \\
(0.921-1.251)\end{array}$ & 0.37 & $\begin{array}{c}0.837 \\
(0.667-1.050)\end{array}$ & 0.06 \\
\hline & & $\mathrm{PM}_{10}$ & $\begin{array}{c}0.951 \\
(0.850-1.064)\end{array}$ & 0.38 & $\begin{array}{c}1.044 \\
(0.933-1.168)\end{array}$ & 0.45 & $\begin{array}{c}0.911 \\
(0.779-1.064)\end{array}$ & 0.12 \\
\hline
\end{tabular}

$\mathrm{PM}_{2.5}$, particulate matter with a diameter of $2.5 \mu \mathrm{m}$ or less; $\mathrm{PM}_{10}$, particulate matter with a diameter of $10 \mu \mathrm{m}$ or less; Temp., temperature.

\section{Discussion}

The majority of studies that take up the matter of air pollution are conducted in big cities, often in Chinese megalopolises and densely populated Indian agglomerations. This leads to the marginalization of the problem in smaller cities. However, if we compare SDRs in Suwałki and Łomża with the mean 
SDR for Poland (1638 and 1945 vs. 1218 per 100,000 inhabitants), we come to the realization that there are no reasons for this negligence [16].

Major findings of the study are the following: in both of the cities, despite differences in air quality, the influence of PMs on total and cardiovascular mortality was observed, while $\mathrm{PM}_{2.5}$ was associated with pulmonary mortality in Łomża. Different lag patterns were observed for each of the cities.

The origin of air pollutants has an impact on the chemicals that are bound on the surface of particulate matter $[17,18]$. When comparing the health effects of various $\mathrm{PM}_{2.5}$ sources, diesel exhaust particles (DEPs) represent the highest mutagenic activity, generate the most intracellular reactive oxygen species (ROS), and cause altered vascular transcription [19-21]. Generation of ROS is crucial and influences both long- and short-term effects of air pollutants.

The burden of diesel exhaust particles was exposed by Yorifuji et al. in their quasi-experimental study. In 2003 in Tokyo, diesel emission control ordinance was introduced, forcing diesel vehicles to meet the norms for PM. It resulted in a spectacular $44 \%$ decrease in $\mathrm{PM}_{2.5}$. Compared to Osaka, which introduced similar regulations in 2009, there was a decrease in cardiovascular mortality of $11 \%$, ischemic heart disease mortality of $10 \%$, and cerebrovascular disease mortality of $6.2 \%$ [22].

The topic of poor air quality in smaller cities is a multi-level issue. Firstly, in both analyzed cities, due to the lack of ring roads, major national trunk roads run through the city centers. Traffic lights, crossroads, pedestrian crossings, and heavy traffic during peak hours extend the time of large goods vehicles spent on passing the city. Secondly, when comparing highly urbanized cities with more rural ones, the higher prevalence of detached houses in the latter is apparent. It has been proved in numerous studies that air pollutants' concentrations fluctuate during the year, reaching the highest values during heating seasons. It seems obvious that its peak is linked with coal combustion processes that are more intensified in housing estates. The third issue is the lack of air quality monitoring stations in smaller cities. In Suwałki, $\mathrm{PM}_{2.5}$ concentration measurements started only in 2014, and in September 2020 there are still no gaseous pollutants concentration measurements stations. Lastly, the favoring of coal-fired power plants by authorities is an important obstacle on the way to the cleaner air.

Łomża represented over twice higher mean daily concentrations of $\mathrm{PM}_{2.5}$ than Suwałki (28.4 vs. $12.6 \mu \mathrm{g} / \mathrm{m}^{3}$ ) and exceeded mean daily WHO guidelines almost five times more frequently ( $40.7 \% \mathrm{vs} .8 .4 \%$ ). When analyzing $\mathrm{PM}_{10}$ concentrations, although differences in values were not so highly demonstrated, they remain higher in Łomża ( $21.7 \mathrm{vs.} 29.0 \mu \mathrm{g} / \mathrm{m}^{3}$ ).

When comparing the contributions of cause-specific mortality in all-cause mortality between two cities, the most apparent disbalance lies in the prevalence of cerebral infarctions. The more polluted city, Łomża demonstrated almost twice higher cerebral infarction mortality rate than Suwałki $(9.2 \%$ vs. $5.8 \%)$. This comes in line with many studies reporting the link between an increase in particulate matter concentrations and the risk of cerebrovascular events [23,24]. Referring to research, $\mathrm{PM}_{2.5}$ has a greater impact on mortality than $\mathrm{PM}_{10}$ [25]. Shi et al. in their analysis of $\mathrm{PM}_{2.5}$-induced premature mortality reported that stroke was the most important cause, accounting for $39 \%$ of the total mortality [26]. The mechanisms of influence are not fully understood yet, however few pathways are considered to be possible. They include endothelial dysfunction, systemic inflammation, rheological parameter alternations, and formation of thrombi as a result of atrial fibrillation induction [27,28].

Many studies from the past provided strong evidence on particulate matter influence on cardiovascular mortality [29,30]. It was reported that an increase in $10 \mu \mathrm{g} / \mathrm{m}^{3}$ of $\mathrm{PM}_{2.5}$ and $\mathrm{PM}_{10}$ concentrations increased daily cardiovascular mortality rate by $0.55 \%$ and $0.36 \%$, respectively [31]. Reported associations were stronger especially in cities with lower annual mean particulate matter concentrations. As we expected, our study proved that PMs have an influence on cardiovascular mortality. $\mathrm{PM}_{2.5}$ was responsible for CVD mortality in both of the analyzed cities. However, $\mathrm{PM}_{10}$ manifested its influence only in less polluted of the analyzed cities (Suwałki). No lag effect was observed.

It is widely acknowledged that $\mathrm{PM}_{2.5}$ is more harmful for the respiratory system than $\mathrm{PM}_{10}$ [32]. Smaller size permits deeper inhalation into small alveoli, resulting in greater surface of interaction. 
Induction of inflammation and generation of ROS seems to be crucial in a detrimental influence on the respiratory system [33]. The most recent hypotheses put in the spotlight the influence of $\mathrm{PM}_{2.5} \mathrm{on}$ airway microbiome profile and alternation of pulmonary function [34]. Interestingly, meta-analysis of $\mathrm{PM}_{2.5}$ and daily mortality revealed that a $10 \mu \mathrm{g} / \mathrm{m}^{3}$ increment was responsible for a higher risk of death due to respiratory rather than cardiovascular causes (1.51\% vs. $0.84 \%$ ) [35]. In our study, IQR increase in exposure to $\mathrm{PM}_{2.5}$ concentrations had an impact on mortality in Łomża only on the day after exposure. Some studies claim that the early lag effect is more specific for cardiovascular diseases, whereas the prolonged lag effect is linked with respiratory diseases, especially asthma [36]. Similar results were reported in a research conducted in Madrid, showing relationship between short-term exposure and increase in all-cause pulmonary mortality on lags 1 and 2 [37].

The effect of IQR increase on $\mathrm{PM}_{2.5}$ concentrations on all-cause mortality was similar in both of the cities, showing a statistically significant association only on the day of exposure. When comparing the results with other European studies, we notice some heterogeneity. It has been proved that $\mathrm{PM}_{2.5}$ have a significant influence on mortality in Stockholm and the Netherlands [38,39]. In the latter study, the influence on all-cause mortality was reported on all of the analyzed lags. On the other hand, Atkinson et al. found little evidence for associations of $\mathrm{PM}_{2.5}$ and all-cause mortality in London [40].

$\mathrm{PM}_{10}$ had an influence on all-cause mortality on lag 1 only in Łomża and in both of the cities on lag 2 . Similar results were reported in several studies. The study by Choi et al. conducted in Seoul proved that $\mathrm{PM}_{10}$ was associated with all-cause and cardiovascular mortalities, however not with respiratory mortalities [41]. The aforementioned Dutch study observed an association between $\mathrm{PM}_{10}$, all cause, and respiratory mortality [39]. Recent Sicilian research proved an association between $\mathrm{PM}_{10}$, all-cause, and cause-specific mortality [42].

A different pattern of lag effects between cities has been reported in other publications [43]. It could be due to different sources of pollution, resulting in different chemicals bound on the surface of particulate matter [44]. Another hypothesis might include differences in gaseous pollutant concentrations, as the amplifying effect of the harmful influence of gaseous pollutants on particulate matter is well-known [45].

In this study, we used data only from patients registered as residents of Łomża and Suwałki. Having only one monitoring station in each of the cities, it made spatiotemporal variations impossible to analyze, leading to unavoidable exposure misclassification. Both of the monitoring stations lie within the boundaries of the cities. Exposure misclassification bias might have been less significant in Łomża, as the city's area is over twice as small as Suwałki's. Yu et al. in their work analyzed the impact of spatiotemporal subject mobility on estimates of ambient air pollutant exposure [46]. They compared home location-based exposure (HBE) and call detail record location-based exposure (CDRE). Mean $\mathrm{PM}_{2.5}$ exposure was only $0.4 \mu \mathrm{g} / \mathrm{m}^{3}$ higher in CDRE $\left(72.9 \mathrm{vs} .72 .5 \mu \mathrm{g} / \mathrm{m}^{3}\right)$. Taking into consideration the results mentioned above, relatively small areas of the analyzed cities, and the fact that nondifferential misclassification of exposure bias is towards the null association, we expect the alternation of results, especially in Suwałki, but not to the extent that would mask the correct conclusions.

\section{Conclusions}

In the whole studied region despite differences in air quality, the influence of PMs on all-cause mortality was observed. This effect was prolonged up to one and two days after the exposure. Cardiovascular mortality in both of the cities was influenced by $\mathrm{PM}_{2.5}$ on lag 0 , whereas $\mathrm{PM}_{10}$ was associated only with higher mortality rate in Suwałki. Pulmonary mortality rate was associated with increase in $\mathrm{PM}_{2.5}$ concentrations only in Łomża on lag 1. Implementation of strategies aimed at reducing exposure to traffic-derived air pollution will contribute to the improvement of cardiovascular and respiratory health. 


\section{Limitations}

The main limitation of our study was the lack of gaseous pollutants data. This information could have let us draw better conclusions concerning lag effects and cause-specific mortality. Having only one monitoring station in each of the cities certainly lead to some exposure misclassification. Lastly, case-specific mortality might be underestimated due to garbage codes.

Author Contributions: Conceptualization, Ł.K. and A.K.; methodology, Ł.K.; formal analysis, Ł.K. and E.J.D.; investigation, A.K. and E.J.D.; data curation, Ł.K.; writing-original draft preparation, Ł.K., A.K. and E.J.D.; writing-review and editing, Ł.K., A.K. and E.J.D.; visualization, E.K.; supervision, S.D. and H.B.-G. All authors have read and agreed to the published version of the manuscript.

Funding: This research received no external funding.

Conflicts of Interest: The authors declare no conflict of interest.

\section{References}

1. Bae, S.; Kwon, H.J. Current State of Research on the Risk of Morbidity and Mortality Associated with Air Pollution in Korea. Yonsei Med. J. 2019, 60, 243-256. [CrossRef] [PubMed]

2. Mokoena, K.K.; Ethan, C.J.; Yu, Y.; Shale, K.; Liu, F. Ambient air pollution and respiratory mortality in Xi'an, China: A time-series analysis. Respir. Res. 2019, 20, 139. [CrossRef]

3. Carugno, M.; Consonni, D.; Randi, G.; Catelan, D.; Grisotto, L.; Bertazzi, P.A.; Biggeri, A.; Baccini, M. Air pollution exposure, cause-specific deaths and hospitalizations in a highly polluted Italian region. Environ. Res. 2016, 147, 415-424. [CrossRef] [PubMed]

4. Sanyal, S.; Rochereau, T.; Maesano, C.N.; Com-Ruelle, L.; Annesi-Maesano, I. Long-Term Effect of Outdoor Air Pollution on Mortality and Morbidity: A 12-Year Follow-Up Study for Metropolitan France. Int. J. Environ. Res. Public Health 2018, 15, 2487. [CrossRef]

5. Anderson, J.O.; Thundiyil, J.G.; Stolbach, A. Clearing the air: A review of the effects of particulate matter air pollution on human health. J. Med. Toxicol. 2012, 8, 166-175. [CrossRef]

6. Jiang, X.Q.; Mei, X.D.; Feng, D. Air pollution and chronic airway diseases: What should people know and do? J. Thorac. Dis. 2016, 8, E31-E40. [CrossRef]

7. Chin, M.T. Basic mechanisms for adverse cardiovascular events associated with air pollution. Heart 2015, 101, 253-256. [CrossRef]

8. Stanaway, J.D.; Afshin, A.; Gakidou, E.; Lim, S.S.; Abate, D.; Abate, K.H.; Abbafati, C.; Abbasi, N.; Abbastabar, H.; Abd-Allah, F.; et al. Global, regional, and national comparative risk assessment of 84 behavioural, environmental and occupational, and metabolic risks or clusters of risks for 195 countries and territories, 1990-2017: A systematic analysis for the Global Burden of Disease Study 2017. Lancet 2018, 392, 1923-1994. [CrossRef]

9. Mills, N.L.; Donaldson, K.; Hadoke, P.W.; A Boon, N.; MacNee, W.; Cassee, F.R.; Sandström, T.; Blomberg, A.; E Newby, D. Adverse cardiovascular effects of air pollution. Nat. Clin. Pract. Cardiovasc. Med. 2009, 6, 36-44. [CrossRef] [PubMed]

10. World Health Organization. Revision of the European Standard Population Report of Eurostat's Task Force. Available online: https://ec.europa.eu/eurostat/documents/3859598/5926869/KS-RA-13-028-EN.PDF/ e713fa79-1add-44e8-b23d-5e8fa09b3f8f (accessed on 1 July 2020).

11. World Health Organization. Ambient (Outdoor) Air Quality and Health (Updated 2 May 2018). Available online: https:/www.who.int/news-room/fact-sheets/detail/ambient-(outdoor)-air-quality-andhealth (accessed on 1 July 2020).

12. Maclure, M. The case-crossover design: A method for studying transient effects on the risk of acute events. Am. J. Epidemiol. 1991, 133, 144-153. [CrossRef]

13. Janes, H.; Sheppard, L.; Lumley, T. Case-crossover analyses of air pollution exposure data: Referent selection strategies and their implications for bias. Epidemiology 2005, 16, 717-726. [CrossRef] [PubMed]

14. Altman, D.G.; Bland, J.M. Statistics Notes Interaction revisited: The difference between two estimates. BMJ 2003, 326, 219. [CrossRef] [PubMed] 
15. Von Elm, E.; Altman, D.G.; Egger, M.; Pocock, S.J.; Gøtzsche, P.C.; Vandenbroucke, J.P. The Strengthening the Reporting of Observational Studies in Epidemiology (STROBE) statement: Guidelines for reporting observational studies. J. Clin. Epidemiol. 2008, 61, 344-349. [CrossRef] [PubMed]

16. Eurostat: Causes and Occurrence of Deaths in the EU. Available online: https://ec.europa.eu/eurostat/web/ products-eurostat-news/-/DDN-20190716-1 (accessed on 5 September 2020).

17. Feng, B.; Li, L.; Xu, H. PM 2.5-bound polycyclic aromatic hydrocarbons (PAHs) in Beijing: Seasonal variations, sources, and risk assessment. J. Environ. Sci. 2019, 77, 11-19. [CrossRef]

18. Al-Naiema, I.M.; Yoon, S.; Wang, Y.Q. Source apportionment of fine particulate matter organic carbon in Shenzhen, China by chemical mass balance and radiocarbon methods. Environ. Pollut. 2018, 240, $34-43$. [CrossRef] [PubMed]

19. Park, C.G.; Cho, H.K.; Shin, H.J. Comparison of Mutagenic Activities of Various Ultra-Fine Particles. Toxicol. Res. 2018, 34, 163-172. [CrossRef]

20. Cho, H.K.; Park, C.G.; Shin, H.J. Comparison of the in vitro toxicological activity of various particulate matter. Toxicol. Ind. Health 2018, 34, 99-109. [CrossRef]

21. Campen, M.J.; Lund, A.K.; Doyle-Eisele, M.L. A comparison of vascular effects from complex and individual air pollutants indicates a role for monoxide gases and volatile hydrocarbons. Environ. Health Perspect. 2010, 118, 921-927. [CrossRef]

22. Yorifuji, T.; Kashima, S.; Doi, H. Fine-particulate Air Pollution from Diesel Emission Control and Mortality Rates in Tokyo: A Quasi-experimental Study. Epidemiology 2016, 27, 769-778. [CrossRef]

23. Wilker, E.H.; Preis, S.R.; Beiser, A.S. Long-term exposure to fine particulate matter, residential proximity to major roads and measures of brain structure. Stroke 2015, 46, 1161-1166. [CrossRef]

24. Zeng, W.; Zhang, Y.; Wang, L. Ambient fine particulate pollution and daily morbidity of stroke in Chengdu, China. PLoS ONE 2018, 13, e0206836. [CrossRef]

25. Zhang, R.; Liu, G.; Jiang, Y. Acute Effects of Particulate Air Pollution on Ischemic Stroke and Hemorrhagic Stroke Mortality. Front. Neurol. 2018, 9, 827. [CrossRef]

26. Shi, Y.; Matsunaga, T.; Yamaguchi, Y. Long-term trends and spatial patterns of PM 2.5-induced premature mortality in South and Southeast Asia from 1999 to 2014. Sci. Total. Environ. 2018, 631-632, 1504-1514. [CrossRef]

27. Kowalska, M.; Kocot, K. Short-term exposure to ambient fine particulate matter (PM2.5 and PM10) and the risk of heart rhythm abnormalities and stroke. Postepy Hig. Med. Dosw. 2016, 70, 1017-1025. [CrossRef]

28. Shah, A.S.; Lee, K.K.; McAllister, D.A. Short term exposure to air pollution and stroke: Systematic review and meta-analysis. BMJ 2015, 350, h1295. [CrossRef]

29. Li, W.; Cao, Y.; Li, R. The spatial variation in the effects of air pollution on cardiovascular mortality in Beijing, China. J. Expo. Sci. Environ. Epidemiol. 2018, 28, 297-304. [CrossRef]

30. Pun, V.C.; Kazemiparkouhi, F.; Manjourides, J. Long-Term PM2.5 Exposure and Respiratory, Cancer, and Cardiovascular Mortality in Older US Adults. Am. J. Epidemiol. 2017, 186, 961-969. [CrossRef]

31. Liu, C.; Chen, R.; Sera, F. Ambient Particulate Air Pollution and Daily Mortality in 652 Cities. N. Engl. J. Med. 2019, 381, 705-715. [CrossRef]

32. Li, M.H.; Fan, L.C.; Mao, B. Short-term Exposure to Ambient Fine Particulate Matter Increases Hospitalizations and Mortality in COPD: A Systematic Review and Meta-analysis. Chest 2016, 149, 447-458. [CrossRef]

33. Xing, Y.F.; Xu, Y.H.; Shi, M.H. The impact of PM2.5 on the human respiratory system. J. Thorac. Dis. 2016, 8, E69-E74. [CrossRef]

34. Wang, L.; Cheng, H.; Wang, D. Airway Microbiome Is Associated with Respiratory Functions and Responses to Ambient Particulate Matter Exposure. Ecotoxicol. Environ. Saf. 2019, 167, 269-277. [CrossRef]

35. Atkinson, R.W.; Kang, S.; Anderson, H.R. Epidemiological time series studies of PM2.5 and daily mortality and hospital admissions: A systematic review and meta-analysis. Thorax 2014, 69, 660-665. [CrossRef]

36. Kim, S.Y.; Peel, J.L.; Hannigan, M.P. The temporal lag structure of short-term associations of fine particulate matter chemical constituents and cardiovascular and respiratory hospitalizations. Environ. Health Perspect. 2012, 120, 1094-1099. [CrossRef] [PubMed]

37. Guaita, R.; Pichiule, M.; Maté, T. Short-term impact of particulate matter $(\operatorname{PM}(2.5))$ on respiratory mortality in Madrid. Int. J. Environ. Health Res. 2011, 21, 260-274. [CrossRef] [PubMed]

38. Meister, K.; Johansson, C.; Forsberg, B. Estimated short-term effects of coarse particles on daily mortality in Stockholm, Sweden. Environ. Health Perspect. 2012, 120, 431-436. [CrossRef] [PubMed] 
39. Janssen, N.A.; Fischer, P.; Marra, M. Short-term effects of PM2.5, PM10 and PM2.5-10 on daily mortality in The Netherlands. Sci. Total. Environ. 2013, 463-464, 20-26. [CrossRef] [PubMed]

40. Atkinson, R.W.; Fuller, G.W.; Anderson, H.R. Urban ambient particle metrics and health: A time-series analysis. Epidemiology 2010, 21, 501-511. [CrossRef]

41. Choi, Y.; Kim, H.; Lee, J.T. Temporal variability of short term effects of PM 10 on mortality in Seoul, Korea. Sci. Total. Environ. 2018, 644, 122-128. [CrossRef]

42. Renzi, M.; Forastiere, F.; Calzolari, R. Short-term effects of desert and non-desert PM 10 on mortality in Sicily, Italy. Environ. Int. 2018, 120, 472-479. [CrossRef]

43. Yoo, S.E.; Park, J.S.; Lee, S.H. Comparison of Short-Term Associations between PM 2.5 Components and Mortality across Six Major Cities in South Korea. Int. J. Environ. Res. Public Health 2019, 16, 2872. [CrossRef]

44. Wang, Y.; Shi, Z.; Shen, F. Associations of daily mortality with short-term exposure to PM2.5 and its constituents in Shanghai, China. Chemosphere 2019, 233, 879-887. [CrossRef]

45. Liu, M.; Xue, X.; Zhou, B. Population susceptibility differences and effects of air pollution on cardiovascular mortality: Epidemiological evidence from a time-series study. Environ. Sci. Pollut. Res. Int. 2019, 26, 15943-15952. [CrossRef] [PubMed]

46. Yu, H.; Russell, A.; Mulholland, J. Using cell phone location to assess misclassification errors in air pollution exposure estimation. Environ. Pollut. 2018, 233, 261-266. [CrossRef] [PubMed]

Publisher's Note: MDPI stays neutral with regard to jurisdictional claims in published maps and institutional affiliations. 